\title{
LA EXPRESIÓN LINGÜÍSTICA DE LA ACTITUD EN EL GÉNERO DE OPINIÓN: EL MODELO DE LA VALORACIÓN
}

\author{
THE LINGUISTIC EXPRESSION OF ATTITUDE IN OPINION \\ DISCOURSE: THE APPRAISAL THEORY
}

\section{MARÍA JOSÉ GONZÁLEZ RODRÍGUEZ \\ Universidad de La Laguna. Tenerife, España. \\ majgonza@ull.es}

\section{RESUMEN}

Dentro del marco de la lingüística sistémico-funcional (Halliday, 1994; Iedema, Fezz y White, 1994; Martin, 2000; White, 2003) la Teoría de la Valoración constituye un instrumento teórico y analítico valioso en el estudio de la evaluación en el lenguaje. Este acercamiento elabora la noción de significado interpersonal y explora, describe y explica el modo en el que el lenguaje se usa para evaluar, adoptar posiciones, construir personas textuales y manejar posturas y relaciones interpersonales. En particular, la Valoración se ocupa de la expresión lingüística de la actitud y la emoción. En este contexto, este trabajo examina los recursos evaluativos en el amplio dominio semántico de la actitud, centrándose en los datos proporcionados por artículos de opinión pertenecientes a periódicos nacionales británicos. Además, este artículo pretende mostrar el papel de los significados evaluativos en la diseminación de la ideología, en la constitución de estilos textuales e identidades del autor y en la negociación de las relaciones escritor/lector.

Palabras clave: Teoría de la Valoración, lenguaje actitudinal, actitud, afecto, juicio, apreciación, evaluación.

\section{ABSTRACT}

Located within the framework of systemic functional linguistics (Halliday, 1994; Iedema, Fezz y White, 1994; Martin, 2000; White, 2003) Appraisal Theory constitutes a valuable theoretical and analytic tool in the study of evaluation in language. This approach elaborates on the notion of interpersonal meaning and explores, describes and explains the way language is used to evaluate, to adopt stances, to construct textual personas and to manage interpersonal positionings and relationships. More particularly, Appraisal is concerned with the language of attitude and emotion. In this context, this work examines evaluative 
resources in the broad semantic domain of attitude, focussing upon the data provided by British national newspapers comment articles. Further, this paper intends to show the role evaluative meanings play in the dissemination of ideology, in the constitution of textual styles and authorial identities, and in the negotiation of writer/reader relationships.

Keywords: Appraisal Theory, attitudinal language, attitude, affect, judgement, appreciation, evaluation.

Recibido: 04/06/2010. Aceptado: 03/03/2011

\section{INTRODUCCIÓN}

T a Teoría de la Valoración (Martin y Rose, 2003; White, 2003; Martin y White, 2005) constituye un desarrollo reciente dentro del estudio de la evaluación en el lenguaje, la actitud y la emoción, así como de los recursos que hacen variar el compromiso del emisor ${ }^{1}$ con sus enunciados. Originada en la Universidad de Sydney, los fundamentos de la Teoría de la Valoración se ubican en el marco de la lingüística sistémico-funcional (Halliday, 2004) y en las nociones de dialogismo y heteroglosia (Bajtín, 1981, 1982, 1999). En este ámbito, la Teoría de la Valoración representa en la actualidad una valiosa herramienta teórica y analítica que posibilita la realización de investigaciones sobre la evaluación en el lenguaje desde una perspectiva integradora.

Son muchos los investigadores que, desde hace décadas, han dedicado su atención al estudio de la evaluación, tanto en el discurso oral (Labov, 1972; Grimes, 1975; Sinclair y Coulthard, 1975) como en el escrito (Hoey, 1983; Fowler, 1991; Tadros, 1994; Winter, 1994; Hunston y Thompson, 2000), con el fin de proponer nuevos desarrollos teóricos que integren diversas perspectivas que suministren una mayor comprensión de los usos evaluativos del lenguaje. Dentro de las posturas más recientes en el estudio de la evaluación destaca la Teoría de la Valoración en el discurso académico, periodístico y legal (Iedema et al., 1994; Martin, 1995a, 1995b, 1997, 2000, 2003, 2004; Christie y Martin, 1997; Coffin, 1997; White, 1998, 2000, 2002, 2003, 2004; Rothery y Stenglin, 2000; Martin y Rose, 2003; Hood, 2004; Miller, 2004; Martin y White 2005, entre otros). También sobresalen estudios relacionados con el fenómeno de la prosodia evaluativa, aspecto importante de la evaluación implícita (Lemke, 1992, 1998). El modelo de la valoración se ha aplicado además al análisis de la conversación (Eggins y Slade, 1997) y de la narrativa (Macken-Horarik, 2003; Page, 2003); a la lingüística computacional (Taboada y Grieve, 2004); al estudio de la adquisición de la lengua

${ }^{1}$ A lo largo de este trabajo, las expresiones emisor, autor, hablante (entre otras similares) se utilizan de manera indistinta. 
materna (Painter, 2003) así como de los textos multimodales (Macken-Horarik, 2004). En el ámbito latino, destacan las investigaciones de Bolívar (1994a, 1994b, 1995, 1996a, b, 1997, 1999, 2001, 2005, 2006), quien se ha interesado por la negociacion de la evaluación en el texto escrito, y en el papel de la evaluación en el diálogo y la confrontación; los trabajos de Shiro (1996a, 1996b, 1997, 1998, 2001, 2003), quien estudia, entre otros aspectos, la relación entre la evaluación y las habilidades narrativas, y los de Erlich (1994, 1995, 1997, 1999, 2000), quien observa el fenómeno evaluativo desde la perspectiva de la argumentación.

Cabe subrayar que la mayoría de los estudios vinculados con el modelo de la valoración han sido publicados en lengua inglesa. De hecho, existen en la actualidad pocos estudios publicados en lengua española, si bien se percibe una influencia creciente de esta teoría en España, Brasil, Chile y Argentina ${ }^{2}$. De cualquier manera, es de destacar que investigadores en Latinoamérica y Argentina están trabajando dentro de este marco. Un texto más o menos reciente el Manual de Lingüistica Sistémico Funcional. El enfoque de M. A. K. Halliday y R. Hasan. Aplicaciones a la lengua española (2005), de Elsa Ghio y M. D. Fernández. Asimismo, pueden señalarse los trabajos de Achugar (1999, 2002, 2004) sobre el género testimonial y los editoriales en periódicos; Gallardo y Ferrari (2006), sobre el discurso científico; Kaplan y Solá (2002), sobre las estrategias retóricas en artículos políticos; además de la publicación en español de Hood y Martin (2005) sobre el papel de la gradación en la evaluación implícita en el discurso académico.

Recientemente, la Teoría de la Valoración ha despertado un gran interés en los investigadores en lingüística y análisis del discurso en diversas lenguas (véase, por ejemplo, el grupo de discusión virtual Appraisal Analysis) ${ }^{3}$, y ha comenzado a aplicarse para estudiar una variedad de aspectos relacionados con el lenguaje evaluativo y la negociación de posiciones intersubjetivas. La investigación de esta teoría se centra particularmente en la comprensión de cómo el uso de diferentes recursos evaluativos puede variar en función del género, registro o estilos individuales, e intenta ahondar en los supuestos ideológicos subyacentes, desvelando las estrategias retóricas mediante las cuales las posturas ideológicas se transforman en naturales. Esta teoría pretende igualmente explicar la forma en que los textos construyen para sí mismos un tipo de interlocutor o lector "ideal” y "no ideal", y descubre por qué algunos textos se interpretan como ambivalentes, ambiguos o inconsistentes desde el punto de vista evaluativo. Además, intenta comprender cómo el uso de diferentes recursos evaluativos en un texto contribuye a estructurarlo como una unidad discursiva.

El estudio que se presenta a lo largo de estas páginas se enmarca dentro de esta

\footnotetext{
2 Para una exposición en español más detallada de los inicios de la Teoría de la Valoración, véase Kaplan (2004) y Ciapuscio (2005).

${ }^{3}$ Appraisal Analysis. Foro de discusión en línea. URL: http://groups.yahoo.com/group/Appraisal Analysis.
} 
corriente, con el propósito de describir y explicar los recursos evaluativos empleados en el discurso de los editoriales en diferentes publicaciones nacionales británicas. En este contexto, el modelo de análisis que se aplica en este estudio pretende poner de relieve aspectos contextuales y culturales, admitiendo la posibilidad de múltiples lecturas de los significados actitudinales, a través de una adaptación del modelo de la valoración al análisis de textos producidos en diferentes ámbitos culturales.

\section{CRITERIOS DE SELECCIÓN DEL CORPUS}

Un aspecto distintivo de la prensa nacional británica es su división en prensa de calidad o de formato sábana y prensa popular o tabloide. El término de calidad se refiere por lo general a aquella prensa de un alto nivel cultural, analítica, detallada y extensa en las noticias, que cuida los textos, seria (sin rozar el sensacionalismo), honesta y que cuenta con credibilidad. Por lo habitual, este tipo de prensa pone énfasis en la información internacional, caracterizándose así por una orientación cosmopolita con el objetivo de que sus lectores puedan obtener una información amplia. Se define por un marcado énfasis en el relato interpretativo, en la política, en las relaciones internacionales, economía, bienestar social, educación, ciencia y cultura. Además, refleja una determinación por servir y ayudar a desarrollar a unos lectores intelectuales y bien educados tanto a nivel nacional como extranjero, destacando también su deseo de llamar la atención e influenciar a los líderes de opinión. Teniendo en cuenta todos estos factores, es comprensible por qué la primera página de un periódico de formato sábana se diseña con el fin de obtener el alcance más amplio de interés.

Por contraste, en la prensa que se define como popular o tabloide se tiende a una mayor personalización de la información, a una redacción segmentada y sencilla, más esquemática que en la prensa de calidad y al empleo de un vocabulario cotidiano, sugerente, emotivo e incisivo. Se especializa en grandes fotografías y en ocasiones en una presentación dramática de la información. Presenta titulares amplios, con frecuencia sensacionalistas que contrastan con la exposición de un relato conciso. Este tipo de prensa está dirigida a aquellos lectores que quieren entretenerse a través de la lectura del periódico al mismo tiempo que informarse.

Atendiendo a esta clasificación, plenamente consolidada en la prensa nacional británica, el presente estudio se ciñe a un género específico, el género de opinión, en diarios nacionales británicos de formato sábana y de formato tabloide. En líneas generales, los artículos de opinión han recibido menos atención teórica y empírica que el relato periodístico informativo (ver, por ejemplo, Van Dijk, 1985, 1986, 1988; Bell, 1991; Fowler, 1991; Scollon y Scollon, 1997; White, 1998; González Rodríguez, 1999, 2001; Ungerer, 2000). Sin embargo, dado que los artículos de opinión constituyen una parte importante en la composición de un 
periódico, éstos han ocupado un lugar destacado en la prensa escrita, en el estudio del texto y en la lingüística discursiva (véase Van Dijk, 1998; Vestergaard, 1999; White, 2003; Martin y White, 2005), así como en el análisis crítico del discurso (Fairclough, 1995; Van Dijk, 2001) y, más recientemente, en la lingüística del corpus y en el análisis histórico (Westin, 2002), entre otros.

La descripción se ilustra con el estudio de una muestra de artículos de opinión tomados de los periódicos The Guardian y The Sun en relación con un tema común, los trágicos sucesos que tuvieron lugar en Norteamérica el 11 de septiembre de 2001 y los acontecimientos posteriores al mismo ${ }^{4}$. Cabe precisar que el estudio que aquí se presenta es principalmente cualitativo, en tanto que mi interés particular se ha centrado en desarrollar la naturaleza de las categorías que se van a analizar e ilustrarlas con ejemplos de discurso real tomado de prensa escrita actual. En lo relativo a la justificación para la elección de los dos diarios objeto de estudio, ésta se ha formulado en base al propósito comunicativo de cada publicación en cuestión. A este respecto, el diario de calidad The Guardian y el popular The Sun pertenecen a idiosincrasias culturales diferentes, se dirigen a diferentes lectores $y$, sobre la base de diferencias en estilo y contenido, estos dos periódicos muestran diferentes intereses y niveles de educación. En relación a la composición del corpus, éste consta de 12 artículos de opinión en cada uno de los dos periódicos en el período comprendido entre el 11 de septiembre y el 31 de octubre de 2001. Dadas las limitaciones de espacio del presente artículo, gran parte de los ejemplos a los que se alude en el análisis se limitan a dos artículos de opinión seleccionados para ilustrar la explicación: Beyond belief (The Guardian, 12 de septiembre de 2001) y Tragic proof (The Sun, 21 de septiembre de 2001)5.

Esta muestra inicial ha sido contrastada con otra muestra posterior de características similares, con el fin de asegurar la validez de los resultados obtenidos en la muestra inicial. Para ello, se constituyó igualmente un corpus de 12 artículos de opinión en cada uno de los periódicos elegidos inicialmente en el estudio (The Guardian y The Sun) en relación a un tema en común, los recientes atentados terroristas en Libia. El análisis contrastivo de los resultados obtenidos de tal estudio se recoge en el apartado 5 de este artículo.

\section{LA TEORÍA DE LA VALORACIÓN: FUNDAMENTOS TEÓRICOS}

La Teoría de la Valoración se ubica en el marco de la lingüística sistémico-funcional desarrollada por Michael Halliday (1975, 1978, 1994, 2004), recogiendo

\footnotetext{
${ }^{4}$ La muestra seleccionada para el estudio (2001 y 2011) pertenece a la versión electrónica de los dos periódicos (www.guardian.co.uk y www.thesun.co.uk). Todas las referencias que se hacen a estos diarios en el análisis pertenecen a los mencionados archivos de la web.

${ }^{5}$ Véase el apéndice de este trabajo, donde se recoge la transcripción de los citados artículos.
} 
la tradición de la lingüística europea y remontándose sus orígenes a la Escuela de Praga. La teoría propuesta por Halliday se caracteriza en líneas generales por su orientación funcional y semántica, poniendo énfasis en la relación entre el lenguaje y el contexto social.

La Teoría de la Valoración se desarrolla a partir del trabajo de investigación en educación iniciado en el Departamento de Lingüística de la Universidad de Sydney bajo la dirección de James R. Martin. El proyecto original (Write it right) formó parte del programa New South Wales Disadvantaged Schools. Los lingüistas que colaboraron con este proyecto, casi todos pertenecientes a la corriente sistémico-funcional, estudiaron los requerimientos necesarios para que los alumnos de la escuela secundaria pudiesen entender los discursos científicos, tecnológicos y mediáticos y, de esta manera, desenvolverse con eficacia en los estudios de historia, literatura inglesa, geografía y artes visuales. Una de las conclusiones a las que llegaron los investigadores fue que, para responder a los numerosos interrogantes surgidos durante el desarrollo del proyecto, era necesaria una comprensión más delicada de la semántica interpersonal. En especial se requería profundizar en el modelo de tenor así como en las consecuencias retóricas y de posicionamiento social asociadas a las diferentes opciones que ofrece la léxico-gramática, y en la necesidad de identificar la solidaridad ideológica o valorativa como un parámetro clave en torno al cual varían los aspectos interpersonales del contexto social.

Para la lingüística sistémico-funcional, el lenguaje es considerado como un recurso sistemático para expresar significados en un contexto. De este modo, el lenguaje es conceptualizado como un sistema de opciones semánticas, de entre las cuales el emisor selecciona las que le permiten transmitir mejor su mensaje al interlocutor o lector. Las opciones disponibles dependen del contexto, y se registran en diferentes niveles del lenguaje: el nivel semántico, el nivel léxico-gramático y el nivel fonológico. El proceso de producción del enunciado (que se inicia con la elección de los significados y culmina con la estructuración de los mismos) se lleva a cabo simultáneamente en tres planos de significado. Estos planos, a los que Halliday denomina metafunciones, son el del contenido (metafunción ideacional), el de la interacción (metafunción interpersonal) y el del texto mismo (metafunción textual). Precisamente, la Teoría de la Valoración centra su interés en profundizar en el estudio de la metafunción interpersonal, y es a través de ésta como se manifiesta la interacción social y se ubica la expresión de nuestros puntos de vista sobre eventos y personas.

El concepto de registro (Halliday, 1978) es de gran importancia para la Teoría de la Valoración. El registro es una explicación teórica de la observación de sentido común que indica que usamos el lenguaje de modos diferentes en situaciones diferentes (Eggins y Martin, 1997: 340). Tres son las variables contextuales que integran el registro y que determinan si el empleo de un género específico en una situación concreta es apropiado o no. Estas variables son campo, tenor y modo. 
1. Campo, la acción social: lo que está pasando, la naturaleza de la acción social que está ocurriendo, es decir, en qué están ocupados los participantes, donde el lenguaje es como un componente esencial.

2. Tenor, la estructura de roles: quién está participando, la naturaleza de los participantes, sus posiciones y roles, es decir, qué tipos de relaciones de rol existen entre los participantes, incluyendo relaciones permanentes y temporales de uno u otro tipo, tanto los tipos de roles discursivos que adoptan en el diálogo como todo el grupo de relaciones socialmente significativas en las que están involucrados.

3. Modo, la organización simbólica: qué papel desempeña el lenguaje, qué es lo que esperan los participantes que el lenguaje haga por ellos en la situación, es decir, la organización simbólica del texto, la posición que éste ocupa y su función en el contexto, incluyendo el canal de expresión (si es hablado, escrito o una combinación de ambos) y también el modo retórico, lo que se logra por medio del texto en términos de categorías como persuasivo, expositivo, didáctico y otros por el estilo.

Los investigadores inmersos en la Teoría de la Valoración se han centrado en la variable contextual de tenor, en particular en la constitución de los roles, las relaciones sociales y la negociación. En particular, Martin (1992) elaboró esta noción e identificó las nociones de poder/estatus, contacto y afecto, a través de las cuales se pueden organizar las relaciones sociales. Asimismo, Poynton $(1985,1989)$ amplió la formulación de Brown y Gilman (1960) en relación con la selección de pronombres en función de las variables de poder y solidaridad, y suministró un modelo de tenor donde distingue, al igual que Martin, las dimensiones de poder/ estatus, contacto y afecto, e identifica los principios de reciprocidad, de proliferación y de amplificación. Sin embargo, los investigadores del proyecto original Write it right consideran que el contacto debe interpretarse en un sentido menos concreto e interactivo que el definido por Poynton, destacando que el factor esencial es la manera en que los textos construyen lo que White (2004) denomina contacto valorativo o ideológico con sus presuntos lectores potenciales. Estos desarrollos sugirieron así que el término solidaridad era preferible al de contacto para este modo de posicionamiento social.

En esencia, el proyecto Write it right reveló la necesidad de comprender mejor las consecuencias retóricas de un valor interpersonal en lugar de otro, y la necesidad de identificar la solidaridad ideológica o valorativa como un parámetro clave en torno al cual varían los aspectos interpersonales del contexto social. La investigación dio lugar a una explicación del juicio evaluativo como un conjunto de significados por medio de los cuales los hablantes valoran el comportamiento de las personas y la apreciación, como un conjunto de significados para establecer valoraciones estéticas y de otro tipo, de los objetos y productos. En otros casos, es- 
tas necesidades dan lugar a nuevos enfoques para establecer modelos de potencial retórico de determinadas opciones léxico-gramaticales y las relaciones que pueden establecerse entre estas opciones.

En síntesis, el enfoque propuesto por la Teoría de la Valoración constituye indudablemente una perspectiva complementaria a los estudios de la lingüística sistémica sobre semántica discursiva interpersonal. Si bien la tradición sistémicofuncional se ha enfocado en el diálogo como intercambio de bienes y servicios, o de información, ha faltado en los análisis un estudio de cómo se sienten los interlocutores, qué juicios emiten y qué valor asignan a los diferentes fenómenos de su experiencia. De ahí la importancia de los sistemas propuestos por la Teoría de la Valoración, que sobrepasan los modelos de intercambio basados en la gramática y toman en consideración estas dimensiones adicionales de la comunicación.

\section{LA ACTITUD EN THE GUARDIANY THE SUN: ANÁLISIS DE LOS DATOS DISCURSIVOS}

Como se ha señalado con anterioridad, la valoración incluye todos los usos evaluativos del lenguaje mediante los cuales los hablantes y escritores adoptan posturas de valor particulares y negocian estas posturas con sus interlocutores reales o potenciales. Esta teoría proporciona así una imagen global de cómo se formula el sistema de valoración y de los tipos de cuestiones semánticas que permite abordar.

La Teoría de la Valoración ofrece un marco para explorar cómo y con qué fines retóricos los hablantes y autores adoptan una postura actitudinal hacia el contenido experiencial de sus enunciados, una postura hacia sus interlocutores reales o potenciales, y una postura hacia la heteroglosia del contexto intertextual en el que operan sus enunciados y textos. En otras palabras, para este enfoque el uso evaluativo del lenguaje tiene como función establecer un posicionamiento actitudinal, un posicionamiento dialógico y un posicionamiento intertextual (White, 2004). La posición actitudinal está vinculada a los significados mediante los cuales los textos/hablantes atribuyen un valor o una evaluación intersubjetiva a los participantes y procesos, ya sean relacionados con respuestas emocionales o con sistemas de valores culturalmente determinados. La posición dialógica tiene que ver con los significados susceptibles de negociación entre los emisores y sus receptores reales o potenciales. En este sentido, todos los enunciados y textos toman en consideración a otros enunciados anteriores o bien reaccionan ante ellos. Finalmente, y por lo que respecta al posicionamiento intertextual, éste se vincula con los usos lingüísticos mediante los cuales los emisores adoptan posturas evaluativas hacia las posiciones representadas como provenientes de fuentes externas, es decir, los puntos de vista y las aseveraciones de otros hablantes y autores. La forma básica 
del posicionamiento intertextual es la que expresa una relevancia implícita, en la que el emisor decide citar o hacer referencia a las palabras o a los pensamientos de otros.

Los recursos evaluativos, según la Teoría de la Valoración, pueden dividirse en tres dominios semánticos: la actitud, el compromiso y la gradación. En la actitud se incluyen los significados por los cuales los textos o hablantes atribuyen un valor o una evaluación intersubjetiva a los participantes y a los procesos. En el dominio del compromiso se incluyen los recursos lingüísticos que pueden utilizarse para posicionar la voz del hablante o del autor en relación con las diversas proposiciones y propuestas comunicadas por un texto. Por último, por medio de la gradación se representa un espacio semántico de escala que está relacionado con la manera en que los hablantes intensifican o disminuyen la fuerza de sus enunciados y gradúan el foco de sus categorizaciones semánticas.

A continuación muestro los resultados de la investigación, fruto de la aplicación del modelo de análisis expuesto a los textos que conforman la muestra. Para acometer este objetivo, nos adentraremos en el estudio de uno de los grandes dominios semánticos en el sistema de la valoración, la actitud, con el fin de interpretar cómo los emisores en The Guardian y The Sun hacen uso de los recursos evaluativos pertenecientes a este eje semántico-discursivo y construyen estratégicamente el discurso en los artículos de opinión, en función de los objetivos del género dentro de su contexto.

\subsection{La actitud}

Se clasifican como actitudinales aquellos enunciados que transmiten una evaluación positiva o negativa, o que pueden interpretarse como una invitación al lector a suministrar sus propias evaluaciones. Esta categoría se divide en los subsistemas de afecto, juicio y apreciación. Mientras que el afecto es la caracterización de los fenómenos en relación con las emociones y puede considerarse como el sistema actitudinal básico, el juicio y la apreciación son formas institucionalizadas de las emociones. Así, el juicio comprende significados que sirven para evaluar positiva o negativamente el comportamiento humano con respecto a las normas sociales institucionalizadas; y la apreciación tiene que ver con la evaluación de objetos, procesos, constructos o textos, en función de principios estéticos y otros sistemas de valor social.

En términos generales, cabe destacar que las evaluaciones de actitud predominantes en la muestra objeto de estudio son las de juicio. Los valores de afecto son también numerosos, mientras que los de apreciación destacan en menor medida. Así, de 438 evaluaciones de actitud identificadas en la muestra analizada de The Guardian, 250 corresponden al juicio, mientras que los valores de afecto son 130, y únicamente 58 evaluaciones corresponden a la apreciación. En el corpus de The 
Sun, de 592 evaluaciones de actitud, 321 corresponden al juicio, 236 al afecto y 35 a la apreciación.

\subsubsection{Afecto}

Los valores de afecto proporcionan uno de los modos más obvios en que el hablante adopta una postura o posición frente a determinados fenómenos, mostrando los recursos por medio de los cuales ese fenómeno lo afectó emocionalmente, o apreciando el fenómeno en términos afectivos. Estas evaluaciones o respuestas emocionales ponen en juego la solidaridad entre el emisor y su audiencia. Los indicadores lingüísticos del afecto pueden ser verbos de emoción que remiten a procesos mentales, adverbios que señalan circunstancias de modo, adjetivos que expresan emoción, y nominalizaciones, esto es, transformaciones de verbos y adjetivos en sustantivos.

Las categorías correspondientes al subsistema de afecto aparecen recogidas en la siguiente tabla, adaptada de Eggins y Slade (1997).

Tabla I. Categorías de afecto: expresiones congruentes

\begin{tabular}{|lll|}
\hline \multirow{2}{*}{ Categoría } & \multicolumn{2}{c|}{ AFECTO } \\
\cline { 2 - 3 } Felicidad/Infelicidad & $\begin{array}{l}\text { Ejemplos positivos } \\
\text { feliz, alegre, jubiloso/a, } \\
\text { optimista }\end{array}$ & $\begin{array}{l}\text { deprimido/a, triste, } \\
\text { miserable, angustiado/a }\end{array}$ \\
\hline Satisfacción/Insatisfacción & $\begin{array}{l}\text { interesado/a, absorto/a, } \\
\text { estar enfrascado/a, gustar }\end{array}$ & $\begin{array}{l}\text { cansado/a, aburrido/a, } \\
\text { exasperado/a, odiar }\end{array}$ \\
\hline Seguridad/Inseguridad & $\begin{array}{l}\text { confiado/a, seguro/a, } \\
\text { tranquilo/a, sereno/a }\end{array}$ & $\begin{array}{l}\text { ansioso/a, preocupado/a, } \\
\text { inseguro/a, intranquilo/a }\end{array}$ \\
\hline
\end{tabular}

El afecto puede clasificarse en autoral (correspondiente a la primera persona) y no-autoral (para la segunda y tercera persona). En el afecto autoral, los hablantes indican cómo han respondido emocionalmente a la persona, cosa, situación o evento que se evalúa, y asumen la responsabilidad de esa evaluación. En el afecto denominado no-autoral se describen las emociones de otros individuos o grupos humanos y no las del autor. En este sentido, cuando los hablantes atribuyen alguna emoción a un actor social, se espera que ello suscite una respuesta favorable o desfavorable hacia éste. Así, White (2004) sostiene que los actores sociales representan tipos institucionalizados y no individuos aislados, por lo que un lector que simpatice con la respuesta emocional atribuida a un cierto tipo social está predispuesto a legitimar la posición que ese tipo social representa. Estos aspectos trasladados al corpus analizado muestran que todos los artículos de opinión se in- 
cluyen en la categoría de afecto autoral. Ello es comprensible si se tiene en cuenta que la práctica discursiva del género periodístico de opinión promueve un modelo de presentación de las noticias donde el objetivo básico es la búsqueda del efecto de subjetividad.

En líneas generales, el análisis comparativo de los artículos de opinión de las dos publicaciones que componen la muestra revela diferencias en lo que respecta a la evaluación de los personajes. Si bien los textos de las dos publicaciones presentan personajes que podríamos denominar como parte de la "población americana", The Guardian muestra al pueblo americano de forma generalizada y en cierto modo abstracto, mientras que The Sun retrata víctimas individuales. Esta diferencia observada tiene que ver con los supuestos que los dos periódicos atribuyen a sus respectivos públicos, concediéndoles relevancia noticiosa a distintos detalles de la información. Así, tanto el emisor de The Guardian como el de The Sun son consecuentes con su ideología, por lo que en la reconstrucción del suceso privilegian el marco interpretativo promovido por los intereses de cada publicación. Como muestra de lo dicho, tómense los dos artículos de opinión recogidos en el apéndice de este trabajo.

En todos los textos de la muestra analizada se observa una fuerte tendencia a evaluar a los terroristas por medio de valores ubicados en el polo negativo del eje de la actitud. Se asignan sentimientos negativos a estos individuos, y se critica y censura su conducta. También se emiten evaluaciones desfavorables sobre el atentado, respecto a su motivo injustificado y su crueldad, entre otros aspectos. Al discriminar por grupo de personajes, se observa que, en las dos publicaciones, los terroristas son los personajes más evaluados negativamente, mientras que las víctimas del atentado se ubican en el polo positivo de la evaluación. La tendencia a la evaluación dramática de los sucesos es más acentuada en los artículos de opinión de The Sun que en los de The Guardian, todo lo cual es, en última instancia, un indicador de los intereses y del propósito comunicativo de ambas publicaciones.

Tanto el emisor de The Guardian como el de The Sun asignan la mayoría de los sentimientos, positivos y negativos, a las víctimas del atentado terrorista. La proporción de valores de afecto ubicados en el polo negativo es en The Guardian notablemente superior a la de los valores positivos. De 130 valores de afecto, únicamente 19 son positivos y los restantes negativos. La distribución de los valores de afecto es como sigue: De los 19 valores positivos, 5 pertenecen al dominio de la felicidad y 14 al de la satisfacción, no habiéndose encontrado valores ubicados en el dominio de la seguridad. Tómese como ilustración el siguiente ejemplo, que refleja los sentimientos de orgullo y de admiración por las víctimas del atentado terrorista: Love was all they had to set against the hatred of their murderers. Por lo que respecta a los 111 valores negativos de afecto, éstos se distribuyen de la siguiente manera: 24 corresponden al dominio de la infelicidad, 8 al de la insatisfacción y 
79 al de la inseguridad. En la categoría más frecuente, la de los sentimientos de inseguridad, $45 \%$ de las expresiones lingüísticas representan la antipatía (un sentimiento dirigido a "los otros", como el odio y el aborrecimiento por el atentado terrorista) y la inseguridad relacionada con la desconfianza en "los otros"; y el 55\% restante corresponde a la tristeza (un sentimiento dirigido "a nosotros", relacionado con el dolor y la frustración por lo acontecido). En lo referido a la insatisfacción, destacan aquellas expresiones que representan sentimientos de displacer, tales como el enojo y la ira. Algunos ejemplos tomados del artículo de opinión de The Guardian recogido en el apéndice ejemplifican lo dicho: If the hijackers had been able to imagine themselves into the thoughts and feelings of the passengers, they would have been unable to proceed; The hijackers used fanatically certainty, misplaced religious faith, and dehumanising hatred to purge themselves of the human instinct for empathy.

La distribución de los valores de afecto en la muestra de The Sun es como sigue: De los 10 valores positivos, 4 corresponden al dominio semántico-discursivo de la felicidad, 3 al de la seguridad, y 3 al de la satisfacción. De los 226 valores negativos, 114 corresponden a la infelicidad, 27 a la insatisfacción y 85 a la inseguridad. En la categoría más frecuente, la de los sentimientos de infelicidad, $74 \%$ de las expresiones lingüísticas representan la tristeza y el restante $26 \%$, la antipatía. Es precisamente la expresión lingüística de infelicidad la que más contribuye en la publicación tabloide a construir discursivamente a las víctimas. En cuanto a la insatisfacción, predominan como en The Guardian las expresiones lingüísticas que representan sentimientos de displacer, tales como el enojo y la ira. La expresión del sentimiento de inseguridad está relacionada con la ansiedad y la preocupación. Al igual que en The Guardian, el emisor en The Sun asigna gran parte de los sentimientos (positivos y negativos) a las víctimas del atentado terrorista. En los siguientes ejemplos puede verse, por una parte, cómo se hace uso del sentimiento de sufrimiento y de tristeza utilizando la estrategia de humanizar a las víctimas, contrastando, por otra parte, con los sentimientos de aborrecimiento hacia los terroristas: She was an innocent victim of evil men with no regard for human life; Our enemies are the men who murdered more than 5,000 people like Sarah Ali.

Como dato significativo, cabe resaltar que en el corpus analizado de The Sun la proporción de valores de afecto es superior que en The Guardian. Esa mayor expresión de afecto es una muestra de la tendencia que se da en la publicación tabloide a humanizar en gran medida a las víctimas y a intensificar notablemente el dramatismo del atentado terrorista. Asimismo, también la densidad de afecto negativo en The Sun es mayor que en The Guardian, lo cual coincide con la línea por parte de The Sun de promover una visión más pesimista del suceso en cuestión, mientras que The Guardian tiende a asignar a los actores del suceso más evaluaciones positivas. 
Una forma muy frecuente de expresar la intensidad emocional es a través del uso del lenguaje figurado, en particular con el empleo de metáforas léxicas. Con respecto a la expresión lingüística de afecto, es notoria la alta proporción de metáforas gramaticales (nominalizaciones de cualidades y de procesos), usadas sobre todo en los artículos de opinión del diario The Guardian, las cuales objetivan los procesos, cualidades o atributos, así como la presencia de metáforas léxicas. Una muestra de ello la constituyen los siguientes ejemplos: our state of fevered astonishment, this stupefied condition, a vast mountain of rubble, sheer amazament, a new mood of exhaustion and despair, the hatred of their murderers, the prerogative of the mighty and venerable, the mechanics of compassion, the banality of these details, a shred of hope, the human instinct of empathy, anguished assertions of love, entre otros. Por contraste, se ha observado que el emisor en los artículos de opinión de The Sun tiende a ilustrar las evaluaciones con ejemplos concretos, detallados y dramatizados, recurriendo a formas léxico-gramaticales congruentes para expresar el afecto, tales como las cualidades (por medio de adjetivos) y los procesos (por medio de verbos), como lo ilustran los siguientes ejemplos: an innocent victim of evil men, his time of great grief, the racist bigotry, emocional face, the state of shock, pervade our minds, cut off, frightened. En definitiva, la dramatización de las emociones en los artículos de opinión publicados en The Sun, con una manipulación emocional marcada y, en consecuencia, con un mayor grado de sensacionalismo en la cobertura del atentado terrorista, contrasta notablemente con la tendencia a la abstracción del afecto en los artículos pertenecientes a The Guardian.

La Figura 1 sintetiza los resultados obtenidos en relación a la distribución de los valores positivos y negativos de afecto en la muestra.

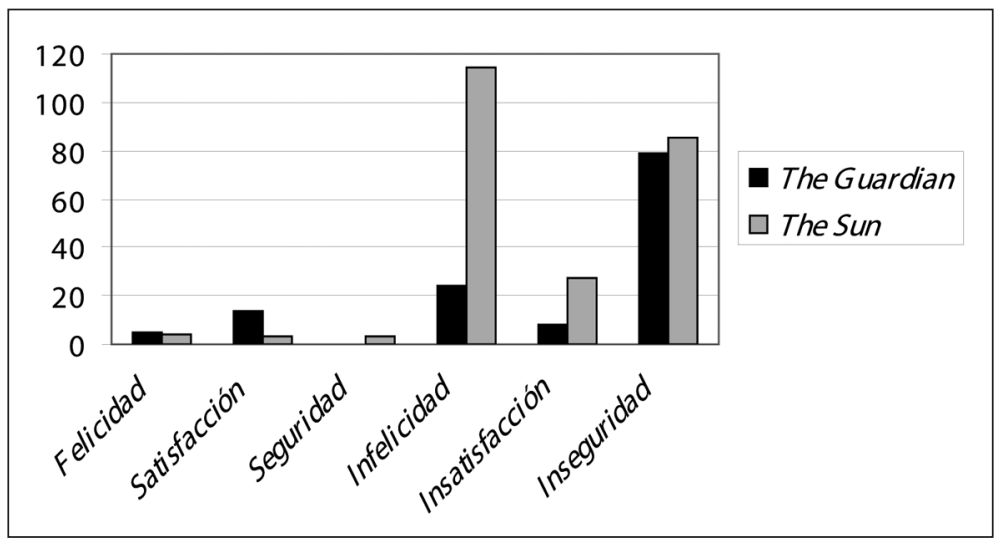

Figura 1. Distribución de valores positivos y negativos de afecto en la muestra. 


\subsubsection{Juicio}

El juicio evaluativo se refiere a la institucionalización de las emociones en el contexto de las propuestas, esto es, normas sobre cómo deben o no deben comportarse las personas. Las normas sociales que se ponen en juego en estas evaluaciones de juicio adoptan la forma de regulaciones o de expectativas sociales. Así, con el juicio se puede evaluar la conducta como moral o inmoral, legal o ilegal, socialmente aceptable o inaceptable, encomiable o deplorable, normal o anormal y así sucesivamente (Iedema, Feez y White 1994; White 1998).

El juicio se clasifica en dos subtipos: juicios de estima social y juicios de sanción social. Los juicios de estima social implican juicios relativos a la normalidad, la capacidad o la tenacidad demostrada en la conducta; es decir, se evalúa lo normal que es una persona, lo competente o resuelta y decidida que es. Los juicios de sanción social implican que está en juego cierto conjunto de reglas o regulaciones más o menos explícitas por la cultura. Esas reglas pueden ser legales o morales, por lo que los juicios de sanción social plantean cuestiones de veracidad y de integridad moral.

La Tabla II, adaptada de Martin (2000), ejemplifica las categorizaciones del subsistema de juicio.

Tabla II. Categorías de juicio: expresiones congruentes.

\begin{tabular}{|c|c|c|}
\hline \multicolumn{3}{|c|}{ JUICIO } \\
\hline & $\begin{array}{l}\text { ESTIMA SOCIAL } \\
\text { Ejemplos positivos } \\
\text { (admiración) }\end{array}$ & $\begin{array}{l}\text { ESTIMA SOCIAL } \\
\text { Ejemplos negativos } \\
\text { (crítica, sin implicaciones legales) }\end{array}$ \\
\hline Normalidad & $\begin{array}{l}\text { Corriente, común, normal, } \\
\text { afortunado/a, moderno/a }\end{array}$ & $\begin{array}{l}\text { Excéntrico/a, extraño/a, raro/a, } \\
\text { desafortunado/a, anticuado/a }\end{array}$ \\
\hline Capacidad & $\begin{array}{l}\text { habilidoso/a, inteligente, } \\
\text { atlético, intuitivo, fuerte }\end{array}$ & inhábil, lento/a, tonto/a, torpe, débil \\
\hline \multirow[t]{2}{*}{ Tenacidad } & $\begin{array}{l}\text { heroico/a, valiente, confiable, } \\
\text { infatigable, perseverante }\end{array}$ & $\begin{array}{l}\text { cobarde, apresurado/a, no confiable, } \\
\text { distraído/a, perezoso/a }\end{array}$ \\
\hline & $\begin{array}{l}\text { SANCIÓN SOCIAL } \\
\text { Ejemplos positivos } \\
\text { (alabanza) }\end{array}$ & $\begin{array}{l}\text { SANCIÓN SOCIAL } \\
\text { Ejemplos negativos (condena, puede } \\
\text { tener implicaciones legales) }\end{array}$ \\
\hline Veracidad & $\begin{array}{l}\text { sincero/a, honesto/a, genuino/a, } \\
\text { franco/a, directo/a }\end{array}$ & $\begin{array}{l}\text { deshonesto/a, manipulador/a, } \\
\text { mentiroso/a, inauténtico/a }\end{array}$ \\
\hline Integridad moral & $\begin{array}{l}\text { moral, bondadoso/a, } \\
\text { respetuoso/a de la ley, sensible, } \\
\text { justo/a }\end{array}$ & $\begin{array}{l}\text { inmoral, malvado/a, corrupto/a, } \\
\text { cruel, injusto/a }\end{array}$ \\
\hline
\end{tabular}


Los juicios pueden expresarse de manera explícita a través de adverbios, atributos y epítetos, sustantivos y verbos. No obstante, en muchas ocasiones la evaluación del juicio puede permanecer implícita, o ser evocada de manera más indirecta mediante los indicadores de juicio. Con éstos, la descripción aparentemente fáctica de un hecho o situación puede desencadenar una evaluación actitudinal. Según White (2004), existe un tercer modo, el juicio provocado, intermedio entre el explícito y el implícito. En esta modalidad se conduce al interlocutor, ya sea mediante fórmulas de contraexpectativa e intensidad, o con el uso de lenguaje evaluativo perteneciente al sistema de afecto, hacia la emisión de un juicio.

El estudio del subsistema juicio en los textos que conforman la muestra nos ha permitido analizar cómo los emisores en The Guardian y The Sun evalúan la conducta de los sujetos que forman parte de la información en base a un marco determinado de normas sociales. Tal y como se subrayó con anterioridad, los valores de juicio son los más frecuentes entre todos los valores de actitud codificados en el corpus. Seguidamente se presentan los resultados del análisis; éstos se refieren a la distribución de valores positivos y negativos de juicios de estima social y de sanción social en la muestra, y en cómo se distribuyen estos valores según los personajes evaluados en las dos publicaciones.

En los artículos de opinión analizados pertenecientes al diario The Guardian hemos contabilizado 250 valores de juicio, de los cuales 195 son negativos y 55 positivos. Los resultados muestran que 172 de esos juicios pertenecen al dominio semántico-discursivo de la sanción social, y 78 juicios al de la estima social. De ello se deduce que el emisor en esta publicación de formato sábana concede mayor importancia a la evaluación de la conducta de los personajes en base a principios éticos codificados en leyes y regulaciones. Por el contrario, los aspectos sociales relativos a las fortalezas o debilidades en su personalidad tienen menos relevancia. El empleo elevado de juicios de sanción social en The Guardian está relacionado con el discurso densamente nominalizado que caracteriza a esta publicación, que despersonaliza a los protagonistas del suceso y que transforma las evaluaciones en presuposiciones, incluso tratándose de artículos de opinión, tal y como queda reflejado en el siguiente ejemplo: Imagining what it is like to be someone other than yourself is at the core of our humanity. It is the essence of compassion, and it is the beginning of morality. Estos juicios de sanción social son en esta publicación mayoritariamente negativos (164 valores negativos frente a 8 positivos).

Sin embargo, con respecto a los juicios de estima social, la proporción de valores positivos y negativos se invierte ya que 47 de estos juicios son positivos y 31 son negativos. Dentro de los valores positivos, predominan (por este orden) la tenacidad y la capacidad, no habiéndose encontrado evaluaciones que juzguen la conducta humana según criterios de normalidad. Dentro de los valores negativos 
de juicio de estima social, el número de evaluaciones negativas con las que se critica a los terroristas por sus acciones supera a los de las otras dos subcategorías. Del total de valores negativos codificados, $55 \%$ corresponde a juicios negativos sobre la capacidad, $39 \%$ a la tenacidad y $6 \%$ a la normalidad. Con respecto a la distribución de los valores de juicio en función del grupo de personajes, las víctimas del atentado son las que reciben mayor número de valores positivos, la mayoría juicios de estima social. En cuanto a los valores negativos, los terroristas y políticos son los más censurados y criticados, desde el punto de vista ético y social. Los personajes comunes reciben muy pocos juicios negativos, con una proporción similar en los de sanción social y estima social. A este respecto, cabe señalar que con los juicios positivos los emisores de The Guardian construyen discursivamente a las víctimas como seres firmes y valientes.

Se ha observado una fuerte tendencia en The Guardian a utilizar metáforas léxicas y otras locuciones figuradas para promover como naturales ciertos juicios sobre el comportamiento de los personajes, o para intensificar la fuerza de las evaluaciones. Ello se ilustra con los siguientes ejemplos: Emotions have their narrative; after the shock we move inevitably to the grief, and the sense that we are doing it more or less together is one tiny scrap of consolation. There was really only one thing for her to say, those three words that all the terrible art, the worst pop songs and movies, the most seductive lies, can somehow never cheapen. I love you.

Por lo que respecta a la muestra analizada de The Sun, hemos contabilizado 321 valores de juicio, de los cuales 118 corresponden a juicios positivos y 203 a valores negativos. De los valores de juicio codificados, 109 pertenecen al dominio de la sanción social y 212 al de la estima social. Ello se traduce en que en los artículos de opinión en The Sun a los juicios que evalúan los aspectos sociales de la conducta y el carácter de los personajes se les asigna mayor importancia que a los morales. De los juicios de sanción social, 102 son negativos y 7 son positivos.

De los juicios positivos, casi todos se corresponden con los valores de integridad moral. Con respecto a los juicios negativos de sanción social, la mayor parte de ellos censura la integridad moral de los terroristas, hecho que coincide con la tendencia observada en la muestra analizada de The Guardian. Al igual que en The Guardian, en los juicios de estima social la proporción de valores positivos y negativos se invierte. De estos últimos, 111 son positivos y 101 son negativos. De los juicios positivos de estima social, el $91 \%$ son valores que elogian la tenacidad de las víctimas y el $9 \%$ su capacidad. Con respecto a los juicios negativos de estima social, las proporciones son similares a las encontradas en The Guardian: el 61\% corresponde a valores de tenacidad, $34 \%$ a valores de capacidad y $5 \%$ a valores de normalidad. Los personajes comunes son los que reciben mayor número de juicios positivos en The Sun. Un rasgo a destacar es cómo las víctimas del atentado terrorista de manera individualizada son evaluadas muy favorablemente en 
la dimensión semántica del juicio como personajes enérgicos, firmes y valientes. Este elevado número de juicios positivos hacia los personajes individuales se produce por una mayor personalización de la información, donde se acumulan muchas evaluaciones favorables hacia las víctimas. Una muestra de ello es el siguiente ejemplo: For Mr Ali is a loyal servant of the Crown. He worked at the Department of Trade and Industry. He loves this country almost as much as he loved his precious daughter. En cuanto a los juicios negativos, los terroristas reciben, al igual que en The Guardian, el mayor número de juicios negativos. En relación con este último punto, cabe destacar una diferencia en lo que concierne a la densidad evaluativa de los juicios negativos en la muestra analizada, en tanto que dicha densidad es más elevada en The Sun que en The Guardian. Véase, a tal efecto, un ejemplo tomado del artículo de opinión de The Sun incluido en el apéndice de este trabajo: In the twisted world of these extremists, the Great Satan, America, is the "enemy" of Islam but they took away the life of one of their own faith.

La Figura 2 muestra la distribución de los juicios positivos y negativos de estima y de sanción social en el corpus analizado.

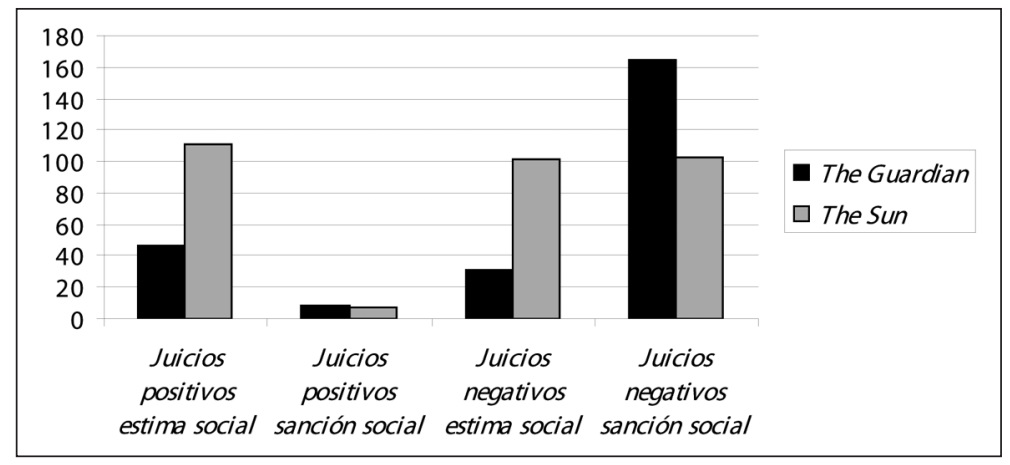

Figura 2. Distribución de juicios positivos y negativos en The Guardian y The Sun.

Finalmente, se mencionan a continuación de forma breve otros aspectos destacados relacionados con el juicio evaluativo, aspectos que se desprenden del estudio realizado. En términos generales, la mayoría de los juicios presentes en los artículos de opinión de los dos diarios analizados pueden inferirse a partir de enunciados con contenido fáctico (White, 1998: 269). Se trata en realidad de versiones del acontecimiento donde el emisor genera principalmente juicios negativos de sanción social con los que censura la conducta, en el caso que nos ocupa, de los terroristas. Por otra parte, se ha comprobado que un modo muy convincente de evaluar la conducta o el carácter de los personajes es a través de determinados 
verbos, particularmente los de valoración negativa, que se emplean para atribuir la palabra a una fuente externa al emisor; de este modo, se consigue evocar juicios negativos de sanción social en relación con la conducta de los causantes del atentado terrorista. Ello se ilustra en el siguiente ejemplo tomado del artículo de The Guardian recogido en el apéndice: The hijackers used fanatical certainty, misplaced religious faith, and dehumanising hatred to purge themselves of the human instict for empathy. Por último, cabe destacar que la intensificación de procesos asociados a determinados individuos es también un recurso utilizado por los emisores de los dos periódicos para provocar juicios sobre el comportamiento de aquéllos. La expresión lingüística de tal intensificación se da a través de adverbios que gradúan en relación con la escala de "vigor" (Hood, 2004). En este sentido, los siguientes ejemplos muestran cómo los adverbios pueden provocar juicios, tanto positivos como negativos, sobre los acontecimientos y los personajes en cuestión: after the shock, we move inevitably to the grief (The Guardian); No other public event had cut so deeply (The Guardian); Astonishingly for a nation so vast, America feels alone (The Sun); But we will never forget them (The Sun).

\subsubsection{Apreciación}

La apreciación se define como el sistema mediante el cual las opiniones y los sentimientos humanos hacia productos, procesos y entidades se institucionalizan como un conjunto de evaluaciones, positivas o negativas. Mientras que el juicio evalúa los comportamientos humanos, la apreciación evalúa artefactos, textos, constructos abstractos tales como planes y políticas, así como objetos naturales o manufacturados. Las personas también pueden ser evaluadas mediante la apreciación, cuando se perciben como entidades y no como participantes con conducta.

Autores como Martin (2000) sostienen que las dimensiones de la apreciación están muy vinculadas a la variable contextual de campo, lo cual se traduce en que la interpretación de este subsistema depende de la posición institucional desde la que se interprete el texto en cuestión. Este autor categoriza la apreciación de acuerdo con tres dimensiones: (1) la reacción, que describe cuánto atrajo nuestra atención el proceso, objeto o texto evaluado y de qué manera nos impactó; (2) la composición, que está relacionada con nuestra percepción de la proporción y el detalle; y (3) la evaluación, que tiene que ver con nuestra apreciación de la importancia social de lo evaluado. La reacción se subdivide en los valores de impacto y calidad, y la composición en los valores de balance y complejidad.

La Tabla III, adaptada de Martin (2000), muestra las categorías y subcategorías de la apreciación. 
Tabla III. Categorías de apreciación: expresiones congruentes.

\begin{tabular}{|lll|}
\hline & \multicolumn{1}{c|}{ Positiva } & \multicolumn{1}{c|}{ Negativa } \\
\cline { 2 - 3 } $\begin{array}{l}\text { REACCIÓN: } \\
\text { Impacto }\end{array}$ & $\begin{array}{l}\text { cautivador/a, llamativo/a, } \\
\text { atractivo/a, agradable, } \\
\text { conmovedor/a }\end{array}$ & $\begin{array}{l}\text { aburrido/a, tedioso/a, } \\
\text { ascético/a, pedante, soso/a }\end{array}$ \\
\hline $\begin{array}{l}\text { REACCIÓN: } \\
\text { Calidad }\end{array}$ & $\begin{array}{l}\text { hermoso/a, espléndido/a, } \\
\text { encantador/a }\end{array}$ & $\begin{array}{l}\text { Feo/a, repulsivo/a, } \\
\text { repugnante }\end{array}$ \\
\hline $\begin{array}{l}\text { COMPOSICIÓN: } \\
\text { Balance }\end{array}$ & $\begin{array}{l}\text { balanceado/a, armonioso/a, } \\
\text { proporcionado/a, simétrico/a }\end{array}$ & $\begin{array}{l}\text { desbalanceado/a, } \\
\text { discordante, } \\
\text { desproporcionado/a, } \\
\text { asimétrico/a }\end{array}$ \\
\hline $\begin{array}{l}\text { COMPOSICIÓN: } \\
\text { Complejidad }\end{array}$ & $\begin{array}{l}\text { simple, elegante, detallado/a, } \\
\text { preciso/a, intrincado/a }\end{array}$ & $\begin{array}{l}\text { extravagante, } \\
\text { monolítico/a, simplista, } \\
\text { impreciso/a }\end{array}$ \\
\hline EVALUACIÓN & $\begin{array}{l}\text { profundo/a, innovador/a, original, } \\
\text { único/a, exigente }\end{array}$ & $\begin{array}{l}\text { superficial, insignificante, } \\
\text { reaccionario/a, } \\
\text { conservador/a }\end{array}$ \\
\hline
\end{tabular}

Del análisis se desprende que la presencia del subsistema de apreciación en los artículos de opinión de The Guardian es exclusivamente negativa, con 58 evaluaciones de apreciación negativas vinculadas todas ellas con el conflicto producido a raíz del atentado terrorista. Dicho conflicto se asocia particularmente con la dimensión de evaluación, ya que recibe 39 de estos valores, relativos a la gravedad, la crueldad y la violencia del atentado terrorista. Los otros valores tienen que ver con la reacción: impacto (11), y con la reacción: calidad (8). Algunos ejemplos obtenidos del corpus son: important, significant, crucial, damaging, dangerous, unhealthy (evaluación); dull, uninviting, arrestable, stunning, unexpected, impressive (reacción: impacto); plain, unattractive, unpleasant, unacceptable (reacción: calidad).

Los resultados del análisis revelan que la presencia de apreciación en los artículos de opinión publicados en The Sun posee también connotaciones negativas, con una proporción menor que en la muestra de The Guardian; en concreto, 35 evaluaciones de apreciación codificadas. La evaluación constituye nuevamente la dimensión más presente en el corpus (28), seguida del valor de reacción: impacto (3), reacción: calidad (2) y composición: balance (2). Este menor porcentaje del subsistema de apreciación en la prensa tabloide es prueba evidente de la tendencia generalizada de este tipo de publicaciones a la subjetividad explícita, alejándose, por lo tanto, de la evaluación de objetos, textos y constructos abstractos, tendencia más propia de la prensa de formato sábana. Tómense como ejemplo algunas de las expresiones de la dimensión de apreciación que se presentan en el corpus, características de la prensa tabloide: dangerous, hard, heavy, strong, enormous, pro- 
found, important, unhealthy (evaluación); tight, sharp, straight (reacción: impacto); horrible, disgusting (reacción: calidad); strange, unpleasant (composición: balance).

Como reflexión final me gustaría destacar la importancia que en el análisis de los valores pertenecientes al sistema de actitud tiene el contexto social y cultural que rodea a las publicaciones nacionales británicas; e igualmente, cómo los emisores del género de opinión emplean como estrategia comunicativa para establecer su postura interpersonal la combinación de la evaluación explícita y la evocada, de modo que el lector de cada publicación identifica y comparte sus interpretaciones. Ello lo hemos visto, en el caso que nos ocupa, con respecto a los indicadores de actitud.

La Figura 3 resume la presencia de las categorías y subcategorías de la apreciación en la muestra analizada.

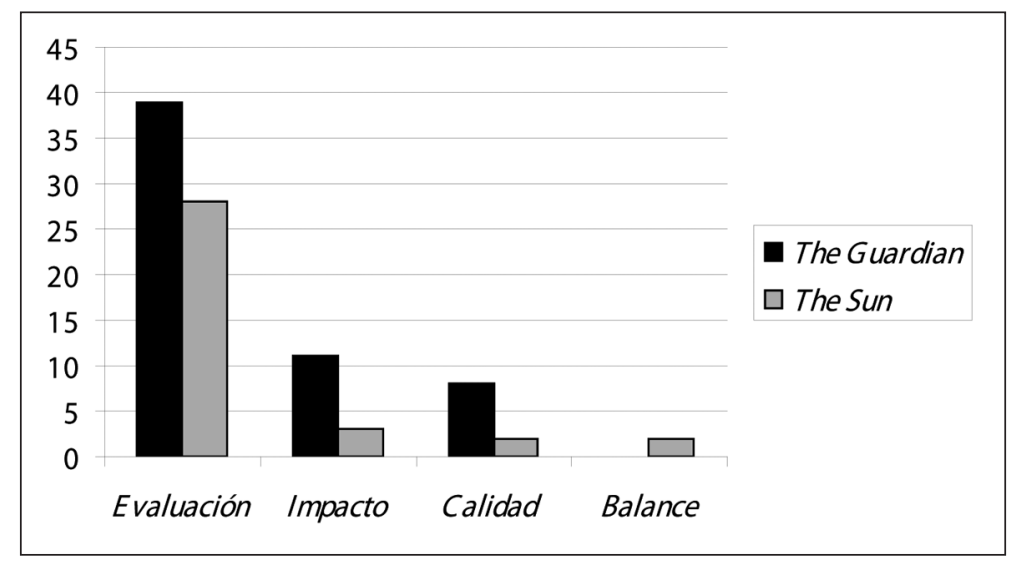

Figura 3. Distribución de las categorías de apreciación negativa en la muestra.

\section{ATENTADOS TERRORISTAS EN LIBIA: ANÁLISIS CONTRASTIVO}

Seguidamente este apartado recoge de forma resumida y a modo de síntesis los resultados del análisis de un corpus de artículos de opinión utilizado en un trabajo reciente (González, 2011), muestra que se ha considerado representativa para el propósito de la presente investigación. Dicha muestra, compuesta por 12 artículos de opinión publicados en The Guardian y The Sun, nos ha permitido llevar a cabo el mismo tipo de análisis que el desarrollado a lo largo de estas páginas, con la finalidad de constatar los datos obtenidos por la muestra inicialmente analizada con artículos de opinión pertenecientes a prensa nacional británica actual; en este caso, informaciones recientes en torno a los atentados terroristas en Libia, cuyas repercusiones en el mundo occidental son evidentes. A este respecto, estos atenta- 
dos terroristas en Libia surgen a partir de un conflicto descrito por los especialistas como un conflicto intenso, prolongado y aparentemente irresoluble. El estudio se ha concentrado en un período breve pero muy violento, durante el cual la cobertura mediática ha sido sumamente intensa (enero-marzo 2011). Ello ha permitido profundizar en el análisis de la construcción discursiva del género de opinión a través de informaciones con implicaciones sociales de creciente gravedad.

En líneas generales, los resultados revelan que, con independencia del intervalo temporal trascurrido entre el análisis de una y otra muestra, se puede establecer una analogía en la distribución del dominio de la actitud y, a su vez, de los subsistemas de afecto, juicio y apreciación, en las publicaciones de los dos mercados, tal y como se recoge a continuación en la Tabla IV. Estos resultados constatan que, dentro de la Teoría de la Valoración, la actitud se refiere a todos aquellos significados que transmiten un valor o una evaluación intersubjetiva de los participantes y de los procesos, valores que pueden estar relacionados tanto con respuestas emocionales (positivas o negativas) como con sistemas de valores culturalmente determinados.

Tabla IV. Evaluaciones de actitud en los atentados terroristas en Libia.

\begin{tabular}{|ccc|}
\hline Valoración: Actitud & The Guardian & The Sun \\
\hline Afecto & 105 & 216 \\
\hline Juicio & 283 & 362 \\
\hline Apreciación & 41 & 37 \\
\hline Total & 429 & 615 \\
\hline
\end{tabular}

A modo de resumen de todos los aspectos aquí tratados, los siguientes ejemplos de los atentados terroristas en Libia ilustran casos patentes de cada uno de los subsistemas de actitud (los valores de los subsistemas están resaltados en negritas):

As a pacifist, I am used to being told that I am naive, cynical or unpatriotic. Most of the media have given me little space to opponents of the bombing of Libya. (The Guardian, 23/03/2011: Libya: Why was pacifism not given a chance?). AFECTO

Targeting Muammar Gaddafi and his military high command is permissible under the broadly drawn terms of the UN Security council resolution, according to many international lawyers. (The Guardian, 21/03/2011: Targeting Gaddafi is allowed by UN resolution). JUICIO 
Unlike Thatcher with her triumphalist 'Rejoice, rejoice' in 1983, the Prime Minister appeared aware of the gravity of the situation as he laid out the justifications for military action in Libya. (The Guardian, 18/03/2011: As David Cameron is drawn into conflict, he is appropriately sombre). APRECIACIÓN

The contents are deemed "extremely reliable". Some 1,000 likely targets in Rome were swiftly given special protection. (The Sun, 24/03/2011: UK terror plot alert). AFECTO

"Time is of the essence. We have seen the uprising of a people against a brutal dictator, and it will send a dreadful signal if their legitimate aspirations are crushed". (The Sun, 15/03/2011: Time to act now over jets). JUICIO

The cupboard is bare. Nobody expects big giveaways. (The Sun, 23/03/2011: Bold comfort). APRECIACIÓN

\section{CONCLUSIÓN}

El objetivo general de la investigación ha sido estudiar la huella del emisor en artículos de opinión referidos a los atentados terroristas a las Torres Gemelas y a los atentados terroristas recientes en Libia, publicados en prensa nacional británica perteneciente a distintos mercados. En el caso que nos ha ocupado, la comparación de la construcción discursiva de estos sucesos en los diarios nacionales británicos The Guardian y The Sun ha mostrado importantes variaciones en la marca del emisor, variaciones que reponden a diferencias estructurales e idiosincrasias de cada publicación en cuestión.

El presente aporte de análisis discursivo permite un estudio integrado dentro de la Teoría de la Valoración en la construcción de los significados interpersonales, a través de las evaluaciones de los personajes involucrados en el evento conflictivo objeto de estudio, la configuración de su rol en el relato y la posterior construcción de marcos interpretativos a través de los valores de actitud. En este sentido, se ha comprobado que los valores de actitud en muy raras ocasiones están presentes de forma explícita en el texto, sino que más frecuentemente pueden ser inferidos a partir de significados experienciales que son los que evocan esas evaluaciones.

En síntesis, a través de este trabajo se ha visto la necesidad de continuar indagando en el estudio del modelo de la Teoría de la Valoración, con el propósito de promover una lectura más crítica de los textos periodísticos, lo cual, en última instancia, nos permitirá reconocer el efecto de la realidad tal y como la producen 
los medios. En relación con este punto, me gustaría terminar haciendo mención a Van Dijk (1995) y a Fowler (1991), cuyos pensamientos resumen en gran medida los aspectos aquí tratados, pensamientos que están relacionados con los esfuerzos llevados a cabo por muchos lingüistas de profundizar en el análisis y en la explicación de cómo se usa el lenguaje para construir significados, y cómo el propio lenguaje se organiza para permitir la construcción de esos significados. Así, Van Dijk (1995: 2) sostiene que: "When expressed in editorials, opinions and ideologies are being produced by journalists and other writers, who exhibit their shared social representations, and participate in the complex processes of newspaper production and reception as well as in intergroup interaction and institutional reproduction". Igualmente, Fowler (1991: 231) en relación con los artículos de opinion afirma que: "The purpose of editorials, therefore, is to emphatically reaffirm this 'truth' for the intended audience. The intended audience uses this point of view, especially in the partisan contexts we have seen, to substantiate its own ideology and, in turn, empower the writers to continue voicing and representing their interests. This process, as we have seen, may differ in content, but its form transcends both culture and ideology."

\section{REFERENCIAS}

Achugar, Mariana. 1999. "Construcción de la memoria: Análisis de la confesión de un represor", en Discurso y Sociedad 4 (1), pp. 7-34.

2002. La construcción de la memoria en el discurso militar Uruguay: 1976-2001. Tesis doctoral no publicada, University of California, Davis, California.

2004. "The events and actors of September 11, 2001 as seen from Uruguay: Analysis of daily newspaper editorials", en Discourse \& Society 15 (2-3), pp. 291-320.

Bajtín, Mijail. 1981. The dialogic imagination: Four essays. Austin, TX: University of Texas Press.

. 1982. Estética de la creación verbal. Primera edición. México: Siglo XXI.

. 1999. An introduction to functional grammar. Segunda edición. London: Edward Arnold.

Bell, Allan. 1991. The language of news media. Oxford: Blackwell.

Bolívar, Adriana. 1994a. Discurso e interacción en el texto escrito. Segunda edición. Caracas: CDCH: Universidad Central de Venezuela.

1994b. "The structure of newspaper editorials". En M. Coulthard (ed.), Advances in Written Text Analysis. London: Routledge, Kegan and Paul, pp. 276-294.

. 1995. "Una metodología para el análisis interaccional del texto escrito", 
en Boletín de Lingüistica 9, pp. 1-18.

. 1996a. "The discourse of British newspaper editorials", en LABSA Journal 1 (1), pp. 75-79.

. 1996b. El control del acceso a la palabra en la noticia periodística. En A. Bolívar (Comp.), Estudios en el Análisis Crítico del Discurso. Cuadernos de Postgrado 14. Caracas: Universidad Central de Venezuela. Comisión de Estudios de Postgrado, pp. 11-45.

1997. "La negociación de la evaluación en editoriales de periódicos", en Boletín de Filología Tomo XXXVI, 7-24. Santiago de Chile: Universidad de Chile, pp. 7-24.

. 1999. "Las metafunciones de la cláusula en español", en Lingua Americana. Año 3 (4), pp. 48-66.

2001. "The negotiation of evaluation in written text". En M. Scott y G. Thompson (eds.), Patterns of Text: In honour of Michael Hoey. Amsterdam: John Benjamins Publishing Company, pp. 129-158.

2005. "Dialogue and confrontation in Venezuelan political interaction", en AILA Review 18, pp. 3-17.

2006. "La función de la evaluación en artículos y ensayos humanísticos". En J. Falk, J. Gille y F. Bermúdez Wachtmeister (Coords.), Discurso, interacción e identidad. Estocolmo: Universidad de Estocolmo, pp. 109-136.

Brown, Roger y Albert Gilman. 1960. "The pronouns of power and solidarity". En Style in language. Ed. Thomas A. Sebeok. Cambridge, Massachusetts: MIT Press, pp. 253-276. Re-editado en Pier Paolo Giglioli (ed.), 1972. Language and social context. Harmondsworth: Penguin, pp. 252-282.

Christie, Frances y James R. Martin (eds.). 1997. Genres and institutions: Social processes in the workplace and school. London: Cassell.

Ciapuscio, Guiomar E. 2005. "La noción de género en la lingüística sistémicofuncional y en la lingüística textual”, en Revista Signos 38 (57), pp. 31-48.

Coffin, Caroline. 1997. "Constructing and giving value to the past: An investigation into secondary school history". En Genres and institutions: Social processes in the workplace and school. Ed. Frances Christie y James R. Martin. London: Cassell, pp. 196-230.

Eggins, Suzanne y Diana Slade. 1997. Analysing casual conversation. London: Cassell.

Eggins, Suzanne y James R. Martin. 1997. "Géneros y registros del discurso". En El discurso como estructura y proceso. Ed. Teun A. van Dijk. Volumen I. Barcelona: Gedisa, pp. 335-371.

Erlich, F. 1994. "La argumentación en el discurso conflictivo: De una reflexión teórica a una actividad pedagógica", en Núcleo 9-10 (EIM, FHE, UCV), pp. 77-82.

.1995. "La autodesignación y la designación de los otros en un periódico 
antirracista francés”, en Núcleo 12 (EIM, FHE, UCV), pp. 25-33.

. 1997. "Implicaciones pragmáticas de los titulares de un periódico antirracista francés", en Lenguaje 25 (Universidad del Valle, Cali, Colombia), pp. 76-86.

1999. Análisis de un periódico francés: macroestructuras semánticas y eficacia discursiva. En Actas del XI Congreso Internacional de la Asociación de Lingüistica y Filología de la América Latina, Las Palmas de Gran Canaria, España, pp. 617-622.

2000. "Las nociones de necesidad y posibilidad en el discurso sobre la Asamblea Constituyente venezolana”, en Núcleo 17, pp. 27-42.

Fairclough, Norman. 1995. Media discourse. London: Edward Arnold.

Fowler, Roger. 1991. Language in the news: Discourse and ideology in the press. London y New York: Routledge.

Gallardo, Susana, y Ferrari, León. 2006. "Estudio diacrónico de la evaluación en las introducciones de artículos científicos de medicina”, en Revista Signos: Estudios de Lingüistica 39 (61), pp. 161-180.

Ghio, Elsa y María Delia Fernández. 2005. Manual de Lingüística Sistémico-Funcional. El enfoque de M. A. K. Halliday y R. Hasan. Aplicaciones a la lengua española. Universidad Nacional del Litorial.

González Rodríguez, María José. 1999. Análisis sociolingüístico-textual del lead en los diarios nacionales británicos. Tesis doctoral. La Laguna: Universidad de La Laguna.

. 2001. "La estructura del relato periodístico informativo en los diarios nacionales británicos”. en Revista Canaria de Estudios Ingleses 42, pp. 289-307.

2011. "La voz del autor en el discurso de opinión: compromiso y solidaridad ideológica", en Revista Canaria de Estudios Ingleses. (En prensa).

Grimes, Joseph. 1975. The thread of discourse. Mouton: The Hague.

Halliday, Michael A.K. 1975. Learning how to mean. London: Edward Arnold. . 1978. Language as social semiotic: The social interpretation of language and meaning. London: Edward Arnold.

. 1994. An introduction to functional grammar. Segunda edición. London: Edward Arnold.

2004. An introduction to functional grammar. Tercera edición. London: Edward Arnold.

Hoey, Michael. 1983. On the surface of discourse. London: George Allen y Unwin.

Hood, Susan. 2004. Appraising research: Taking a stance in academic writing. Tesis doctoral. Sidney: Universidad de Sidney.

Hood, Susan y James R. Martin. 2005. "Invocación de actitudes: El juego de la gradación de la valoración en el discurso", en Revista Signos 38 (58), pp. 195-220.

Hunston, Susan y GeoffThompson. 2000. Evaluation in text: Authorial stance and the construction of discourse. Oxford: Oxford University Press. 
Iedema, Rick, Susan Feez y Peter White. 1994. Media literacy. Sydney: Disadvantaged Schools Program, NSW Department of School Education.

Kaplan, Nora. 2004. "Nuevos desarrollos en el estudio de la evaluación en el lenguaje: La teoría de la valoración”, en Boletín de Lingüistica 22, pp. 52-78.

Kaplan, Nora y Silvia Solá. 2002. "Las estrategias políticas en el discurso de Noam Chomsky", en Akademos 4 (2), pp. 63-82.

Labov, William. 1972. Language in the inner city: Studies in the Black English vernacular. Philadelphia: University of Pennsylvania Press.

Lemke, Jay L. 1992. "Interpersonal meaning in discourse: Value orientations". En Selected papers in Systemic Linguistics. Eds. M. Davies y L. Ravelli. London: Pinter, pp. 82-104.

. 1998. "Resources for attitudinal meaning: Evaluative orientations in text semantics", en Functions of Language 5 (1), pp. 33-56.

Macken-Horarik, Mary. 2003. "Appraisal and the special instructiveness of narrative”, en Text, 23 (2), pp. 285-312.

. 2004. "Interacting with the multimodal text: Reflections on image and verbiage in ArtExpress", en Visual Communication 3 (1), pp. 5-26.

Martin, James R. 1992. English text: System and structure. Amsterdam: John Benjamins.

1995a. "Interpersonal meaning, persuasion, and public discourse: Packing semiotic punch", en Australian Journal of Linguistics 15, pp. 33-67.

. 1995b. "Reading positions/positioning readers: Judgement in English", en Prospect: A Journal of Australian TESOL 10 (2), pp. 27-37.

1997. "Analysing genre: Functional parameters". En Genres and institutions: Social processes in the workplace and school. Eds. Frances Christie y James R. Martin. London: Cassell, pp. 3-39.

2000. "Beyond exchange: Appraisal systems in English". En Evaluation in text: Authorial stance and the construction of discourse. Eds. Susan Hunston y Geoff Thompson. Oxford: Oxford University Press, pp. 142-175.

2003. "Introduction. Negotiating heteroglossia: Social perspectives on evaluation". Text 23 (2), pp. 1-11.

2004. "Mourning: How we get aligned", en Discourse and Society 15 (2-3), pp. 321-344.

Martin, James R. y David Rose. 2003. Working with discourse: Meaning beyond the clause. London: Continuum.

Martin, James R. y Peter White. 2005. The language of evaluation: Appraisal in English. Hampshire y New York: Palgrave Macmillan.

Miller, Donna R. 2004. "Truth, justice and the American way: The Appraisal system of judgement in the U.S. House debate on the impeachment of the President, 1998". En Cross-cultural perspectives on parliamentary discourse. Ed. P. Bayley. Amsterdam y Philadelphia: John Benjamins, pp. 271-300. 
Page, Ruth E. 2003. "An analysis of Appraisal in childbirth narratives with special consideration of gender and storytelling style", en Text, 23 (2): 211-237.

Painter, Clare. 2003. "Developing attitude: An ontogenetic perspective on Appraisal”, en Text, 23 (2), pp. 183-209.

Poyton, Cate. 1985. Language and gender: Making the difference. (Primera edición). Geelong, Vic.: Deakin University Press.

1989. Language and gender: Making the difference. (Segunda edición). Geelong, Vic.: Deakin University Press.

Rothery, Joan y Maree Stenglin. 2000. "Interpreting literature: The role of appraisal". En Researching language in schools and communities: Functional linguistic approaches. Ed. L. Unsworth. London: Cassell, pp. 222-244.

Scollon, Ronald y Suzie W. Scollon. 1997. "Point of view and citation: Fourteen Chinese and English versions of the 'same' news story", en Text 17 (1), pp. 83-125.

Shiro, Martha. 1996a. "Un estudio de las expresiones de modalidad en hablantes de dos culturas", en Boletín de Lingüistica 10, pp. 43-60.

. 1996b. "Venezuelan children's preliterate narrative development: A multidimensional approach”. En A. Stringfellow, D. Cahana-Amitay, E. Hughes y A. Zukowski (eds.), Proceedings of the 20 th annual Boston University Conference on Language Development MA, EE.UU: Cascadilla Press, pp. 706-717.

. 1997. "Labov's model of analysis as an emerging study in discourse", en Journal of Narrative and Life History 7 (1/4), pp. 309-314.

. 1998. Los pequeños cuentacuentos. El desarrollo de las habilidades narrativas de niños en edad escolar. Trabajo de Ascenso Inédito.

2001. "Las habilidades evaluativas en dos tipos de discurso narrativo infantil", en Lingüistica 13, pp. 217-248.

. 2003. "Genre and evaluation in narrative development", en Journal of Child Language 30, pp. 165-195.

Sinclair, John y Malcolm Coulthard. 1975. Towards an analysis of discourse. Cambridge: Cambridge University Press.

Taboada, Maite y Jack Grieve. 2004. "Analyzing Appraisal automatically”. En American association for artificial intelligence spring symposium on exploring attitude and affect in text. Palo Alto, CA: Universidad de Stanford, pp.158-161.

Tadros, Angela. 1994. "Predictive categories in expository prose". En Advances in written text analysis. Ed. M. Coulthard. London: Routledge, pp. 69-82.

Ungerer, Friedrich (ed.). 2000. English Media Texts, Past and Present. Language and textual structure. Amsterdam: Benjamins.

Van Dijk, Teun A. 1985. "Structures of news in the press". En Discourse and communication. New approaches to the analysis of mass media discourse and communication. Ed. Teun A. van Dijk. Berlin and New York: Walter de Gruyter, pp. 69-93. 
. 1986. "News schemata". En Studying writing: Linguistic approaches. Eds. C.R. Cooper y S. Greenbaum. Beberly Hills, California: Sage Publications, pp. 155-185.

. 1988. News as discourse. Hillsdale, NJ: Erlbaum.

. 1995. "Opinions and ideologies in editorials". Unpublished paper for the $4^{\text {th }}$ international symposium of critical discourse analysis. Language, social life and critical thought. Athens, 14-16 diciembre, 1995.

1998. Ideology: A multidisciplinary approach. London: Sage.

.2001. "Critical discourse analysis". En The handbook of discourse analysis.

Eds. D. Schiffrin, D. Tannen y H.E. Hamilton. Malden, Mass: Blackwell, pp. 352-371.

Vestergaard, Torben (ed.). 1999. Language, culture and identity. Aalborg, DK: Aalborg University Press.

Westin, Ingrid. 2002. Language change in English newspaper editorials. Amsterdam: Rodopi.

White, Peter R. 1998. Telling media tales: The news story as rhetoric. Tesis doctoral no publicada. Sidney: Universidad de Sidney.

. 2000. "Dialogue and inter-subjectivity: Reinterpreting the semantics of modality and hedging". En Dialogue analysis VII: Working with dialogue. Eds. Malcolm Coulthard, Janet Cotterill y Frances Rock. Selected papers from the $7^{\text {th }}$ international association of dialogue analysis conference. Birmingham (1999). Tübingen: Neimeyer, pp. 67-80.

. 2002. "Appraisal: The language of evaluation and stance". En The handbook of pragmatics. Eds. Jef Verschueren, Jan-Ola Östman, Jan Blommaert y Chris Bulcaen. Amsterdam/Philadelphia: Benjamins, pp. 1-23.

2003. "Beyond modality and hedging: A dialogic view of the language of intersubjective stance", en Text 23 (2), pp. 259-284.

. 2004. "The language of attitude, arguability and interpersonal positioning".

[En línea]. Disponible en http://www.grammatics.com/appraisal/index.html. [Consulta: 06/05/2010].

Winter, Eugene. 1994. "Clause relations as information structure: Two basic structures in English". En Advances in written text analysis. Ed. Malcolm Coulthard. London: Routledge, pp. 45-78. 


\section{APÉNDICE 1}

\section{The Guardian}

Only love and then oblivion. Love was all they had to set against their murderers Special report: Terrorism in the US

- Ian McEwan

- The Guardian, Saturday 15 September 200116.31 BST

Emotions have their narrative; after the shock we move inevitably to the grief, and the sense that we are doing it more or less together is one tiny scrap of consolation.

Initially, the visual impact of the scenes - those towers collapsing with malign majesty - extended our state of fevered astonishment. Even on Wednesday, fresh video footage froze us in this stupefied condition, and denied us our profounder feelings: the first plane disappearing into the side of the tower as cleanly as a posted letter; the couple jumping into the void, hand in hand; a solitary figure falling with a strangely extended arm (was it an umbrella serving as a hopeful parachute?); the rescue workers crawling about at the foot of a vast mountain of rubble.

In our delirium, most of us wanted to talk. We babbled, by email, on the phone, around kitchen tables. We knew there was a greater reckoning ahead, but we could not quite feel it yet. Sheer amazement kept getting in the way.

The reckoning, of course, was with the personal. By Thursday I noticed among friends, and in TV and radio commentaries, a new mood of exhaustion and despair. People spoke of being depressed. No other public event had cut so deeply. The spectacle was over. Now we were hearing from the bereaved. Each individual death is an explosion in itself, wrecking the lives of those nearest. We were beginning to grasp the human cost. This was what it was always really about.

The silent relatives grouped around the entrances to hospitals or wandering the streets with their photographs was a terrible sight. It reminded us of other tragedies, of wars and natural disasters around the world. But Manhattan is one of the most sophisticated cities in the world, and there were some uniquely modern elements to this nightmare that bound us closer to it.

The mobile phone has inserted itself into every crevice of our daily lives. Now, in catastrophe, if there is time enough, it is there in our dying moments. All through 
Thursday we heard from the bereaved how they took those last calls. Whatever the immediate circumstances, what was striking was what they had in common. A new technology has shown us an ancient, human universal.

A San Francisco husband slept through his wife's call from the World Trade Centre. The tower was burning around her, and she was speaking on her mobile phone. She left her last message to him on the answering machine. A TV station played it to us, while it showed the husband standing there listening. Somehow, he was able to bear hearing it again. We heard her tell him through her sobbing that there was no escape for her. The building was on fire and there was no way down the stairs. She was calling to say goodbye. There was really only one thing for her to say, those three words that all the terrible art, the worst pop songs and movies, the most seductive lies, can somehow never cheapen. I love you.

She said it over and again before the line went dead. And that is what they were all saying down their phones, from the hijacked planes and the burning towers. There is only love, and then oblivion. Love was all they had to set against the hatred of their murderers.

Last words placed in the public domain were once the prerogative of the mighty and venerable - Henry James, Nelson, Goethe - recorded, and perhaps sometimes edited for posterity, by relatives at the bedside. The effect was often consolatory, showing acceptance, or even transcendence in the face of death. They set us an example. But these last words spoken down mobile phones, reported to us by the bereaved, are both more haunting and true.

They compel us to imagine ourselves into that moment. What would we say? Now we know.

Most of us have had no active role to play in these terrible events. We simply watch the television, read the papers, turn on the radio again. Listening to the analysts and pundits is soothing to some extent. Expertise is reassuring. And the derided profession of journalism can rise quite nobly, and with immense resource, to public tragedy.

However, I suspect that in between times, when we are not consuming news, the majority of us are not meditating on recent foreign policy failures, or geopolitical strategy, or the operational range of helicopter gunships.

Instead, we remember what we have seen, and we daydream helplessly. Lately, most of us have inhabited the space between the terrible actuality and these 
daydreams. Waking before dawn, going about our business during the day, we fantasize ourselves into the events. What if it was me?

This is the nature of empathy, to think oneself into the minds of others. These are the mechanics of compassion: you are under the bedclothes, unable to sleep, and you are crouching in the brushed-steel lavatory at the rear of the plane, whispering a final message to your loved one. There is only that one thing to say, and you say it. All else is pointless. You have very little time before some holy fool, who believes in his place in eternity, kicks in the door, slaps your head and orders you back to your seat. 23C. Here is your seat belt. There is the magazine you were reading before it all began.

The banality of these details might overwhelm you. If you are not already panicking, you are clinging to a shred of hope that the captain, who spoke with such authority as the plane pushed back from the stand, will rise from the floor, his throat uncut, to take the controls...

If the hijackers had been able to imagine themselves into the thoughts and feelings of the passengers, they would have been unable to proceed. It is hard to be cruel once you permit yourself to enter the mind of your victim. Imagining what it is like to be someone other than yourself is at the core of our humanity. It is the essence of compassion, and it is the beginning of morality.

The hijackers used fanatical certainty, misplaced religious faith, and dehumanising hatred to purge themselves of the human instinct for empathy. Among their crimes was a failure of the imagination. As for their victims in the planes and in the towers, in their terror they would not have felt it at the time, but those snatched and anguished assertions of love were their defiance. 


\section{APÉNDICE 2}

\section{The Sun}

\section{Tragic proof}

Published: 21 Sep 2001

\section{ADD YOUR COMMENTS}

THE beautiful girl on Page One is tragic proof that the terrorists are not fighting for Islam.

THE beautiful girl on Page One is tragic proof that the terrorists are not fighting for Islam.
Tragically, his daughter is gone now.
Sarah Ali was a British Muslim, who was married to an American. Like everyone on the four hijacked planes and all those at the World Trade Center and the Pentagon, she was an innocent victim of evil men with no regard for human life.

In the twisted world of these extremists, The Great Satan, America, is the "enemy" of Islam? but they took away the life of one of their own faith.

And probably many more, too. Sarah's grieving father, Syed Ali, demonstrates to us all that Britain has nothing to fear from the millions of decent Muslims who live and work here.

For Mr Ali is a loyal servant of the Crown. He worked at the Department of Trade and Industry.

He loves this country almost as much as he loved his precious daughter.

\section{Tragically, his daughter is gone now.}

The Britain he chose to be his home, and which he has served so well, now has a duty to help him in his time of great grief.

It goes without saying that our hearts go out to him. But that is not enough. We must not allow the racist bigotry of those who have turned on British Muslims to pervade our minds. 
We must stand firm in our genuine belief that we have no quarrel with the Islamic faith.

But we will never forget them. Or any of the others who died.

Our enemies are the men who murdered more than 5,000 people like Sarah Ali.

\section{True friend}

NEW Yorkers will never forget Tony Blair. It is impossible to explain what his visit means to them.

Astonishingly for a nation so vast, America feels alone.

Cut off. Frightened.

Britain is the one old friend America can rely on. And when we are needed, we are there for them.

Blair embodies everything that Britain feels for America.

His stunned, emotional face reflected the state of shock we are all still coming to terms with.

When he says "America is our closest ally and friend" and pledges that the links between our two peoples have been further strengthened over the past week, he is speaking for us all.

But yesterday in New York the PM was also opening his heart to the families of the hundreds of Britons killed in the attack.

Many of them are so far unnamed and most of their bodies may never be found.

But we will never forget them. Or any of the others who died. 